\title{
Acid-functionalized Mesoporous Silicate (KIT-5-Pr- SO3H) Synthesized as an Efficient and Nanocatalyst for Green Multicomponent
}

\author{
Mansoureh Daraie, Razieh Mirsafaei, Majid M. Heravi \\ 1- Department of Chemistry, School of Science, Alzahra University, Vanak, Tehran, Iran \\ 2- Department of Pharmaceutics, School of Pharmacy, Isfahan University of Medical Sciences, \\ Isfahan, Iran
}

\begin{abstract}
:
Aim and Objective: KIT-5 nanoporous silica was functionalized with sulfonic acid and $\mathrm{SO} 3 \mathrm{H}$ group has been immobilized on nanoporous KIT-5 silica support via in situ method to produce novel nanocatalyst as "KIT-5-Pr-SO3H". The catalyst was fully characterized by FT-IR, SEM, EDXs, TEM, BET and TGA techniques. The surface morphology images approved that the nanocatalyst particle sizes are around 7-15 $\mathrm{nm}$. The prepared catalyst was efficiently used in the synthesis of benzimidazolo quinazolinones, imidazo[1,2a]chromeno[4,3-d]pyrimidinone and imidazo[1,2-a]pyrimidine via a multicomponent reaction under green conditions. The easy synthesis condition, environmental compatibility, high specific surface area, reusability for 5 run without loses in any activity, high selectivity, availability of raw material, are the remarkable properties for this new catalyst.
\end{abstract}

Materials and Methods: All reagents were purchased from Aldrich and Merck with high-grade quality and used as received. The structural characteristics of the KIT-5 which was obtained, using three-dimensional large cage type face- 
Source: Daraie M, Mirsafaei R, Heravi MM. Acid-functionalized mesoporous silicate (KIT-5-Pr-SO3H) synthesized as an efficient and nanocatalyst for green multicomponent. Curr Org Synth. 2019;16(1):145-53. DOI : $10.2174 / 1570179415666181005110543$

centered cubic Fm3m mesoporous silica materials (KIT-5) nanocages were obtained according to the procedure described by Kleitz et al.

Results: The purpose of this study is developing a new acid-functionalized mesoporous catalyst. Initially, (KIT-5) nanocages were obtained according to the procedure described by Kleitz et al. Then, KIT-5-Pr-SH was prepared by Mercaptopropyltriethoxysilane as illustrated in Scheme 1. In the next step, the solid product was oxidized with $\mathrm{H} 2 \mathrm{O} 2$.

The full characterization for proving the structure of the nano-size particles was achieved using FT-IR, TGA, TEM, SEM, and EDX analysis.

Conclusion: Acid-functionalized mesoporous silica has been proved to act as an effective catalyst in various organic reactions. In this project, for the first time, KIT-5 was functionalized by propyl-sulfonic acid as a heterogeneous solid acid catalyst. Sulfonic acid functionalized KIT-5 (KIT-5-Pr-SO3H) performs as an organicinorganic hybrid catalyst, whereas Brønsted acid sites have been selectively generated. In this regard, the catalytic activities of this novel heterogeneous catalyst were successfully examined by the one-pot multicomponent reaction.

Keywords: Mesoporous supporter, heterogeneous nanocatalyst, acidfunctionalized catalyst, multi-component reaction, green chemistry. 